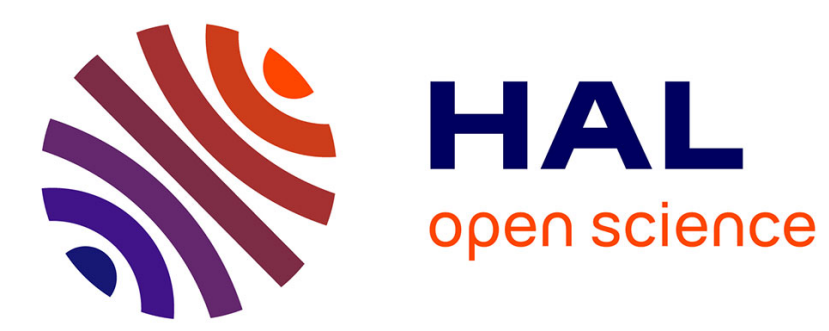

\title{
On the perturbation of the electromagnetic energy due to the presence of small inhomogeneities
}

Christian Daveau, Abdessatar Khelifi

\section{To cite this version:}

Christian Daveau, Abdessatar Khelifi. On the perturbation of the electromagnetic energy due to the presence of small inhomogeneities. 2007. hal-00150590

\author{
HAL Id: hal-00150590 \\ https://hal.science/hal-00150590
}

Preprint submitted on 30 May 2007

HAL is a multi-disciplinary open access archive for the deposit and dissemination of scientific research documents, whether they are published or not. The documents may come from teaching and research institutions in France or abroad, or from public or private research centers.
L'archive ouverte pluridisciplinaire $\mathbf{H A L}$, est destinée au dépôt et à la diffusion de documents scientifiques de niveau recherche, publiés ou non, émanant des établissements d'enseignement et de recherche français ou étrangers, des laboratoires publics ou privés. 


\title{
On the perturbation of the electromagnetic energy due to the presence of inhomogeneities with small diameters
}

\author{
Christian DAVEAU Abdessatar KHELIFI
}

\begin{abstract}
We consider solutions to the time-harmonic Maxwell problem in $\mathbf{R}^{3}$. For such solution we provide a rigorous derivation of the asymptotic expansions in the practically interesting situation, where a finite number of inhomogeneities of small diameter are imbedded in the entire space. Then, we describe the behavior of the electromagnetic energy caused by the presence of these inhomogeneities.
\end{abstract}

\section{Sur la perturbation de l'énergie électromagnétique due à la présence des inhomogénéités de petits diamètres}

\section{Résumé}

Nous considérons des solutions du problème harmonique de Maxwell dans $\mathbf{R}^{3}$. pour une telle solution nous obtenons des formules asymptotiques rigoureuses qui sont dues à la présence d'un nombre fini d'inhomogénéités avec petit diamètre dans l'espace entier. Puis, nous décrivons le comportement de l'énergie électromagnétique provoqué par la présence de ces inhomogénéités.

\section{Version française abrégée}

Dans cette Note, on suppose que dans $\mathbf{R}^{3}$ on a $m$ inhomogénéités $\left\{z_{j}+\alpha B_{j}\right\}_{j=1}^{m}$, où $\alpha$ est un petit paramètre, $B_{j} \subset \mathbf{R}^{3}$ est un ouvert borné et les points $\left\{z_{j}\right\}_{j=1}^{m}$ vérifient l'hypothèse (1). Soient la perméabilité magnétique $\mu_{\alpha}$ et la permittivité électrique $\varepsilon_{\alpha}$ de forme (2).

L'objectif du travail décrit dans cette Note est de voir comment les solutions perturbées du probleme (3) se comportent quand un nombre fini d'inhomogénéités $\left\{z_{j}+\alpha B_{j}\right\}$ de petits diamètres sont introduites dans l'espace entier $\mathbf{R}^{3}$. Ceci nous amène, également, à étudier l'évolution de l'énergie électromagnétique correspondante selon cette déformation du milieu de propagation.

Nous commenons notre analyse dans la section 2 en dérivant rigoureusement les développements asymptotiques des champs électrique et magnétique ce qui sont uniformément validés dans l'espace. Ces formules asymptotiques sont construites par la méthode de développements asymptotiques assorties. Concernant cette méthode, le lecteur peut consulter [8, 11]. En domaine borné, on peut voir aussi les travaux [1, 3, 田, 司. Le terme d'ordre principale, dans des déveoppements asymptotiques analogues (mais pour le cas d'un domaine borné), a été dérivé par Vogelius et Volkov [15] et Ammari et al. [6]. Nos formules asymptotiques utilisent des tenseurs de polarisation associés à des inhomogénéités électromagnétiques qui semblent être des généralisations normales des tenseurs qui ont été présentés par Schiffer et Szegö [14] et complètement étudié par beaucoup d'autres auteurs [2, 7, 9, 12].

Le plan de cette Note est comme suit:

Dans la section 1, Nous formulons le problème principale dans ce travail. Dans la section 2, supposant que les champs électromagnétiques $\left(\mathbf{E}_{\epsilon}, \mathbf{H}_{\epsilon}\right)$ sont dans des espaces de Sobolev convenables, nous obtenons à travers la formule de représentation intégrale de Lippman-Schwinger 
des développements asymptotiques uniformes pour les champs électromagnétiques et la densité d'énergie. Puis, nous formulons une estimation pour l'énergie électromagnétique ce qui nous apporte aux résultats de stabilité néanmoins la présence de petites inhomogénéités.

\section{Problem formulation}

We suppose that there is a finite number of electromagnetic inclusions in $\mathbf{R}^{3}$, each of the form $z_{j}+\alpha B_{j}$, where $B_{j} \subset \mathbf{R}^{3}$ is a bounded and $\mathcal{C}^{\infty}$-domain containing the origin. The total collection of inhomogeneities is:

$$
\mathcal{B}_{\alpha}=\cup_{j=1}^{m}\left\{z_{j}+\alpha B_{j}\right\}
$$

The points $z_{j} \in \mathbf{R}^{3}, j=1, \ldots, m$, which determine the location of the inhomogeneities, are assumed to satisfy the following inequality:

$$
\left|z_{j}-z_{l}\right| \geq c_{0}>0, \forall j \neq l .
$$

Let $\mu_{0}$ and $\varepsilon_{0}$ denote the permeability and the permittivity of the free space, $\omega>0$ is a given frequency and $k=\omega \sqrt{\varepsilon_{0} \mu_{0}}>0$ is the wave number. We denote by $x=\left(x_{1}, x_{2}, x_{3}\right)$ the cartesian coordinates in $\mathbf{R}^{3}$. We shall assume that $\mu_{0}>0$ and $\varepsilon_{0}>0$ are positive constants. Let $\mu_{j}>0$ and $\varepsilon_{j}>0$ denote the permeability and the complex permittivity of the $j$-th inhomogeneity, $z_{j}+\alpha B_{j}$; these are also assumed to be positive constants. Introduce the piecewise-constant magnetic permeability

$$
\mu_{\alpha}(x):= \begin{cases}\mu_{0}, & x \in \mathbf{R}^{3} \backslash \overline{\mathcal{B}}_{\alpha} \\ \mu_{j}, & x \in z_{j}+\alpha B_{j}, j=1 \ldots m .\end{cases}
$$

The piecewise-constant electric permittivity $\varepsilon_{\alpha}(x)$ is defined analogously. If we allow the degenerate case $\alpha=0$, then the function $\mu_{0}(x)$ (resp. $\varepsilon_{0}(x)$ ) equals the constant $\mu_{0}$ (resp. $\varepsilon_{0}$ ).

We suppose now that the collection of inclusions $\mathcal{B}_{\alpha}$ is within an open and fictive subset $\Omega \subset \mathbf{R}^{3}$ and the source current density $\mathbf{J}_{s}$ is at position in $\mathbf{R}^{3} \backslash \bar{\Omega}$.

Let $\left(\mathbf{E}_{\alpha}, \mathbf{H}_{\alpha}\right) \in \mathbf{R}^{3} \times \mathbf{R}^{3}$ denote the time-harmonic electromagnetic fields in the presence of the electromagnetic inclusions $\mathcal{B}_{\alpha}$. These time-harmonic fields [10, 13] are the solutions of the Maxwell's equations

$$
\begin{cases}\nabla \times \mathbf{E}_{\alpha}=i \omega \mu_{\alpha} \mathbf{H}_{\alpha}, & \text { in } \mathbf{R}^{3}, \\ \nabla \times \mathbf{H}_{\alpha}=-i \omega \varepsilon_{\alpha} \mathbf{E}_{\alpha}+\mathbf{J}_{s}, & \text { in } \mathbf{R}^{3}, \\ \nu \times \mathbf{E}_{\alpha} \text { and } \nu \times \mathbf{H}_{\alpha} & \text { are continuous across } \partial\left(z_{j}+\alpha B_{j}\right), \\ \lim _{|x| \rightarrow \infty}|x|\left[\nabla \times \mathbf{E}_{\alpha}-i k \frac{x}{|x|} \times \mathbf{E}_{\alpha}\right]=0 & \text { and } \lim _{|x| \rightarrow \infty}|x|\left[\nabla \times \mathbf{H}_{\alpha}-i k \frac{x}{|x|} \times \mathbf{H}_{\alpha}\right]=0 .\end{cases}
$$

Here $\nu$ denotes the outward unit normal to $\partial\left(z_{j}+\alpha B_{j}\right)$. We eliminate the magnetic field from the above equations by dividing the first equation in (3) by $\mu_{\alpha}$ and taking the curl to obtain the following system of equations for $\mathbf{E}_{\alpha}$ :

$$
\begin{cases}\nabla \times \mu_{\alpha}^{-1} \nabla \times \mathbf{E}_{\alpha}-\omega^{2} \varepsilon_{\alpha} \mathbf{E}_{\alpha}=i \omega \mathbf{J}_{s}, & \text { in } \mathbf{R}^{3}, \\ \nu \times \mathbf{E}_{\alpha} & \text { is continuous across } \partial\left(z_{j}+\alpha B_{j}\right), \\ \lim _{|x| \rightarrow \infty}|x|\left[\nabla \times \mathbf{E}_{\alpha}-i k \frac{x}{|x|} \times \mathbf{E}_{\alpha}\right]=0 . & \end{cases}
$$

Having found the electric field $\mathbf{E}_{\alpha}$, we then obtain the magnetic field $\mathbf{H}_{\alpha}$ through the formula

$$
\mathbf{H}_{\alpha}=\frac{1}{i \omega \mu_{\alpha}} \nabla \times \mathbf{E}_{\alpha} .
$$


The electromagnetic energy is defined by:

$$
\mathcal{E}_{\alpha}(t):=\frac{1}{2} \int_{\mathbf{R}^{3}}\left(\varepsilon_{\alpha}\left|\mathbf{E}_{\alpha}(x, t)\right|^{2}+\left(\mu_{\alpha}\right)^{-1}\left|\mathbf{H}_{\alpha}(x, t)\right|^{2}\right) d x, \quad \text { for } t \geq 0,
$$

and it is not hard to see that $\mathcal{E} \in \mathcal{C}^{1}\left(\mathbf{R}^{+}\right)$.

The finiteness of the electromagnetic energy $\mathcal{E}_{\alpha}$ requires that both the electric and the magnetic field belongs to a space of fields with square integrable curls:

$$
H\left(\operatorname{curl} ; \mathbf{R}^{3}\right):=\left\{\mathbf{a} \in L^{2}\left(\mathbf{R}^{3}\right)^{3} ; \quad \operatorname{curl} \mathbf{a} \in L^{2}\left(\mathbf{R}^{3}\right)^{3}\right\} .
$$

It can be shown that there exists a unique solution $\mathbf{E}_{\alpha} \in \mathcal{C}^{1}\left(\mathbf{R}^{+} ; L^{2}\left(\mathbf{R}^{3}\right)\right) \cap\left(\mathcal{C}^{0}\left(\mathbf{R}^{+} ; H\left(\operatorname{curl} ; \mathbf{R}^{3}\right)\right)\right)$ to the problem (14), and this solution satisfies the following Lippman-Schwinger integral representation formula.

Lemma 1.1 Let $\mathbf{E}_{\alpha}$ be the solution of the problem (团). Then, the following integral representation formula holds

$$
\begin{aligned}
\mathbf{E}_{\alpha}(x)=\mathbf{E}_{0}(x) & +\sum_{j=1}^{m} \int_{z_{j}+\alpha B_{j}}\left(-i \omega\left(\mu_{j}-\mu_{0}\right) \nabla^{\prime} \times \mathcal{G}\left(x, x^{\prime}\right) \cdot \mathbf{H}_{\alpha}\left(x^{\prime}\right)\right. \\
& \left.+\omega^{2} \mu_{0}\left(\varepsilon_{j}-\varepsilon_{0}\right) \mathcal{G}\left(x, x^{\prime}\right) \cdot \mathbf{E}_{\alpha}\left(x^{\prime}\right)\right) d x^{\prime} .
\end{aligned}
$$

The $3 \times 3$ matrix valued function $\mathcal{G}$ means the Green's function solution to

$$
\left\{\begin{array}{l}
\nabla \times \nabla \times \mathcal{G}\left(x, x^{\prime}\right)-k^{2} \mathcal{G}\left(x, x^{\prime}\right)=\mathcal{I}_{3} \delta\left(x-x^{\prime}\right), \quad \text { in } \mathbf{R}^{3} \\
\lim _{|x| \rightarrow \infty}|x|\left[\nabla \times \mathcal{G}\left(x, x^{\prime}\right)-i k \frac{x}{|x|} \times \mathcal{G}\left(x, x^{\prime}\right)\right]=0 .
\end{array}\right.
$$

where $\mathcal{I}_{3}$ is the $3 \times 3$ identity matrix. In the above notation the curl operator, $\nabla \times$, acts on matrices column by column and $\nabla^{\prime}$ denotes the derivation with respect to the second variable $x^{\prime}$.

The proof of Lemma 1.1 follows immediately if we multiply the first equation in (4) by $\mathcal{G}\left(x, x^{\prime}\right) \cdot \mathbf{v}$ $\left(\mathbf{v} \in \mathbf{R}^{3}\right)$ and if we integrate by parts over the domain $\Omega$ which contains the set of inclusions $\left\{z_{j}+\alpha B_{j}\right\}_{j=1}^{m}$.

\section{Asymptotic behavior}

As we said in the introduction our analysis will be dependent on polarization tensors $M \in \mathbf{R}^{3 \times 3}$. We remember that this tensors are defined by

$$
M\left(q_{j} / q_{0} ; B_{j}\right):=\int_{B_{j}} \nabla v^{q}(x) d x,
$$

where $\left\{q_{j}\right\}$ (resp. $q_{0}$ ) denote either the set $\left\{\varepsilon_{j}\right\}$ or $\left\{\mu_{j}\right\}$ for $1 \leq j \leq m$ (resp. denote either $\varepsilon_{0}$ or $\mu_{0}$ ) and where the functions $v^{q}$, which dependent on the contrast $q_{j} / q_{0}$, are solutions of the following problem,

$$
\begin{cases}\nabla \cdot q(x) \nabla v^{q}(x)=0, & \text { in } \mathbf{R}^{3} \\ v^{q}(x)-x \rightarrow 0 & \text { as } x \rightarrow \infty\end{cases}
$$

The function $q$ is given by

$$
q(x)= \begin{cases}q_{0}, & x \in \mathbf{R}^{3} \backslash \overline{B_{j}}, \\ q_{j}, & x \in B_{j} .\end{cases}
$$

The following holds.

Proposition 2.1 Suppose that (1) and (1) are satisfied and let $\left(\mathbf{E}_{\alpha}, \mathbf{H}_{\alpha}\right)$ be the solution of the

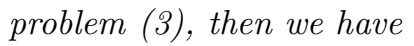


(i) The electric field $\mathbf{E}_{\alpha}$ satisfy the following uniform expansion

$$
\begin{aligned}
\mathbf{E}_{\alpha}(x)= & \mathbf{E}_{0}(x)+\alpha^{3} \sum_{j=1}^{m}\left[-i \omega\left(\mu_{j}-\mu_{0}\right) \nabla^{\prime} \times \mathcal{G}\left(x, z_{j}\right) \cdot \mathbf{M}\left(\mu_{j} / \mu_{0} ; B_{j}\right) \mathbf{H}_{0}\left(z_{j}\right)\right. \\
& \left.+\omega^{2} \mu_{0}\left(\varepsilon_{j}-\varepsilon_{0}\right) \mathcal{G}\left(x, z_{j}\right) \cdot \mathbf{M}\left(\varepsilon_{j} / \varepsilon_{0} ; B_{j}\right) \mathbf{E}_{0}\left(z_{j}\right)\right]+O\left(\alpha^{4}\right) .
\end{aligned}
$$

(ii) The magnetic field $\mathbf{H}_{\alpha}$ satisfy the following uniform expansion

$$
\begin{aligned}
\mathbf{H}_{\alpha}(x) & =\mathbf{H}_{0}(x)+\alpha^{3} \sum_{j=1}^{m}\left[i \omega\left(\varepsilon_{j}-\varepsilon_{0}\right) \nabla^{\prime} \times \mathcal{G}\left(x, z_{j}\right) \cdot \mathbf{M}\left(\varepsilon_{j} / \varepsilon_{0} ; B_{j}\right) \mathbf{E}_{0}\left(z_{j}\right)\right. \\
& \left.-\omega^{2} \varepsilon_{0}\left(\mu_{j}-\mu_{0}\right) \mathcal{G}\left(x, z_{j}\right) \cdot \mathbf{M}\left(\mu_{j} / \mu_{0} ; B_{j}\right) \mathbf{H}_{0}\left(z_{j}\right)\right]+O\left(\alpha^{4}\right) .
\end{aligned}
$$

To evaluate the influence of the presence of these inhomogeneities with small diameters on the evolution of the electromagnetic energy defined by (5), we shall introduce its density per unit of volume which denoted by $\aleph_{\alpha}$. According to Poynting's theorem, energy density $\aleph_{\alpha}$ is given by:

$$
-\frac{\partial}{\partial t} \aleph_{\alpha}=\nabla \cdot \mathbf{\Pi}_{\alpha}+\mathbf{J}_{s} \cdot \mathbf{E}_{\alpha}
$$

where $\boldsymbol{\Pi}_{\alpha}$ is the Poynting vector,

$$
\boldsymbol{\Pi}_{\alpha}=\frac{\mathbf{E}_{\alpha} \times \mathbf{H}_{\alpha}}{\mu_{0}} .
$$

The following theorem is concerned with an important result which justifies the behavior of the electromagnetic energy when a finite number of inhomogeneities are introduced in the entire space.

Theorem 2.1 Suppose that (1) and (11) are satisfied and suppose that the inclusion $B_{j}$ is a ball for each $j \in\{1, \cdots, m\}$. Then, the following uniform expansion holds

$$
\begin{aligned}
\frac{\partial}{\partial t}\left(\aleph_{0}(x, t)-\aleph_{\alpha}(x, t)\right)= & \alpha^{3} \sum_{j=1}^{m} 3\left|B_{j}\right|\left(\frac { \varepsilon _ { j } - \varepsilon _ { 0 } } { \varepsilon _ { j } + 2 \varepsilon _ { 0 } } \left[k^{2} \mathbf{J}_{s} \cdot\left(\mathcal{G}\left(x, z_{j}\right) \cdot \mathbf{E}_{0}\left(z_{j}\right)\right)\right.\right. \\
& +1 / \mu_{0} \nabla \cdot\left[i \omega \varepsilon_{0} \mathbf{E}_{0} \times\left(\nabla^{\prime} \times \mathcal{G}\left(x, z_{j}\right) \cdot \mathbf{E}_{0}\left(z_{j}\right)\right)\right. \\
& \left.\left.+k^{2}\left(\mathcal{G}\left(x, z_{j}\right) \cdot \mathbf{E}_{0}\left(z_{j}\right)\right) \times \mathbf{H}_{0}\left(z_{j}\right)\right]\right] \\
& -\frac{\mu_{j}-\mu_{0}}{\mu_{j}+2 \mu_{0}}\left[i \omega \mu_{0} \mathbf{J}_{s} \cdot\left(\nabla^{\prime} \times \mathcal{G}\left(x, z_{j}\right) \cdot \mathbf{H}_{0}\left(z_{j}\right)\right)\right. \\
& +1 / \mu_{0} \nabla \cdot\left[i \omega \mu_{0}\left(\nabla^{\prime} \times \mathcal{G}\left(x, z_{j}\right) \cdot \mathbf{H}_{0}\left(z_{j}\right)\right)\right. \\
& \left.\left.\left.+k^{2} \mathbf{E}_{0} \times \mathcal{G}\left(x, z_{j}\right) \cdot \mathbf{H}_{0}\left(z_{j}\right)\right]\right]\right)+O\left(\alpha^{5}\right) .
\end{aligned}
$$

Proof.

Recall the following identity: $\nabla \cdot(A \times B)=B \cdot \nabla \times A-A \cdot \nabla \times B$. Relation (12) immediately gives

$$
\nabla \cdot\left(\boldsymbol{\Pi}_{\alpha}\right)=\frac{1}{\mu_{0}}\left[\mathbf{H}_{\alpha} \cdot \nabla \times \mathbf{E}_{\alpha}-\mathbf{E}_{\alpha} \cdot \nabla \times \mathbf{H}_{\alpha}\right]
$$

Next, under assumption that inclusion $B_{j}$ is a ball for all $j \in\{1, \cdots, m\}$, it was proved in [7] that polarization tensors (7) are simplified

$$
M\left(\mu_{j} / \mu_{0} ; B_{j}\right)=\frac{3 \mu_{0}}{\mu_{j}+2 \mu_{0}}\left|B_{j}\right| \mathcal{I}_{3},
$$

and 


$$
M\left(\varepsilon_{j} / \varepsilon_{0} ; B_{j}\right)=\frac{3 \varepsilon_{0}}{\varepsilon_{j}+2 \varepsilon_{0}}\left|B_{j}\right| \mathcal{I}_{3} .
$$

In other words, the inclusions $B_{j}$ are symmetric about their centers (balls). Then, according to [5] the correction of order four in relation (9) is zero and therefore the remainder is in fact $O\left(\alpha^{5}\right)$. Using this result and inserting relations (14) and (15) into relation (9), the following expansions immediately holds:

$$
\begin{gathered}
\mathbf{E}_{\alpha}(x)=\mathbf{E}_{0}(x)+\alpha^{3} \sum_{j=1}^{m}\left[-i \omega \mu_{0} \frac{3\left(\mu_{j}-\mu_{0}\right)}{\left.\mu_{j}+2 \mu_{0}\right)}\left|B_{j}\right| \nabla^{\prime} \times \mathcal{G}\left(x, z_{j}\right) \cdot \mathbf{H}_{0}\left(z_{j}\right)\right. \\
\left.+k^{2} \frac{3\left(\varepsilon_{j}-\varepsilon_{0}\right)}{\left.\varepsilon_{j}+2 \varepsilon_{0}\right)}\left|B_{j}\right| \mathcal{G}\left(x, z_{j}\right) \cdot \mathbf{E}_{0}\left(z_{j}\right)\right]+O\left(\alpha^{5}\right)
\end{gathered}
$$

In similar fashion we can get an expansion for the magnetic field:

$$
\begin{aligned}
\mathbf{H}_{\alpha}(x)= & \mathbf{H}_{0}(x)+\alpha^{3} \sum_{j=1}^{m}\left[i \omega \varepsilon_{0} \frac{3\left(\varepsilon_{j}-\varepsilon_{0}\right)}{\left.\varepsilon_{j}+2 \varepsilon_{0}\right)}\left|B_{j}\right| \nabla^{\prime} \times \mathcal{G}\left(x, z_{j}\right) \cdot \mathbf{E}_{0}\left(z_{j}\right)\right. \\
& \left.-k^{2} \frac{3\left(\mu_{j}-\mu_{0}\right)}{\left.\mu_{j}+2 \mu_{0}\right)}\left|B_{j}\right| \mathcal{G}\left(x, z_{j}\right) \cdot \mathbf{H}_{0}\left(z_{j}\right)\right]+O\left(\alpha^{5}\right) .
\end{aligned}
$$

Using relations (16) and (17), the following holds

$$
\begin{aligned}
\mathbf{H}_{\alpha} \cdot \nabla \times \mathbf{E}_{\alpha}= & \mathbf{H}_{0} \cdot \nabla \times \mathbf{E}_{0}+\alpha^{3}\left\{\sum_{j=1}^{m} c_{1} \mathbf{H}_{0} \cdot \nabla \times\left(\nabla^{\prime} \times \mathcal{G}\left(x, z_{j}\right) \mathbf{H}_{0}\right)\right. \\
& +c_{2} \mathbf{H}_{0} \cdot \nabla \times\left(\mathcal{G}\left(x, z_{j}\right) \mathbf{E}_{0}\right)+\sum_{j=1}^{m} c_{1}^{\prime}\left(\nabla^{\prime} \times \mathcal{G}\left(x, z_{j}\right) \mathbf{E}_{0}\right) \cdot\left(\nabla \times \mathbf{E}_{0}\right) \\
& \left.+c_{2}^{\prime}\left(\mathcal{G}\left(x, z_{j}\right) \mathbf{H}_{0}\right) \cdot\left(\nabla \times \mathbf{E}_{0}\right)\right\}+O\left(\alpha^{5}\right),
\end{aligned}
$$

where the constants $c_{1}, c_{2}, c_{1}^{\prime}$ and $c_{2}^{\prime}$ are given by

$$
\left\{\begin{array}{l}
\frac{c_{1}}{i \omega \mu_{0}}=\frac{c_{2}^{\prime}}{k^{2}}=-3 \frac{\mu_{j}-\mu_{0}}{\mu_{j}+2 \mu_{0}}\left|B_{j}\right|, \\
\frac{c_{2}}{k^{2}}=\frac{c_{1}^{\prime}}{i \omega \varepsilon_{0}}=3 \frac{\varepsilon_{j}-\varepsilon_{0}}{\varepsilon_{j}+2 \varepsilon_{0}}\left|B_{j}\right| .
\end{array}\right.
$$

In the same manner we find the relation for the term $\mathbf{E}_{\alpha} \cdot \nabla \times \mathbf{H}_{\alpha}$; therefore relation (13) becomes

$$
\begin{aligned}
\nabla \cdot\left(\boldsymbol{\Pi}_{\alpha}\right)= & \nabla \cdot\left(\boldsymbol{\Pi}_{0}\right)+\alpha^{3} \sum_{j=1}^{m} \frac{1}{\mu_{0}}\left[c_{1} \nabla \cdot\left(\left(\nabla^{\prime} \times \mathcal{G}\left(x, z_{j}\right) \mathbf{H}_{0}\right) \times \mathbf{H}_{0}\right)\right. \\
& +c_{2} \nabla \cdot\left(\mathcal{G}\left(x, z_{j}\right) \mathbf{E}_{0} \times \mathbf{H}_{0}\right)+c_{1}^{\prime} \nabla \cdot\left(\mathbf{E}_{0} \times\left(\nabla^{\prime} \times \mathcal{G}\left(x, z_{j}\right) \mathbf{E}_{0}\right)\right) \\
& \left.+c_{2}^{\prime} \nabla \cdot\left(\mathbf{E}_{0} \times \mathcal{G}\left(x, z_{j}\right) \mathbf{H}_{0}\right)\right]+O\left(\alpha^{5}\right) .
\end{aligned}
$$

The proof is achieved by expanding the term $\mathbf{J}_{s} \cdot \mathbf{E}_{\alpha}$ at order 5 according to $\alpha$ in (11).

Based on Theorem 2.1, we can prove the following main result.

Theorem 2.2 Let $T>0$, then there exists some positive constant $C$ such that the following estimate as $\alpha \rightarrow 0$ holds uniformly in $t \in[0, T]$

$$
\left|\mathcal{E}_{\alpha}(t)-\mathcal{E}_{0}(t)\right| \leq C \alpha^{3},
$$

where the constant $C$ is independent of $\alpha$ and the set of points $\left\{z_{j}\right\}_{j=1}^{m}$ provided that assumption (17) holds, but this constant $C$ is dependent on $\left|B_{j}\right|$ and $T$. 


\section{References}

[1] H. Ammari, E. IAkovleva, And D. Lesselier, Two numerical methods for recovering small inclusions from the scattering amplitude at a fixed frequency, SIAM J. Sci. Comput., 27 (2005), 130-158.

[2] H. Ammari, AND H. KANG, Reconstruction of small inhomogeneities from boundary measurements, Lecture Notes in Mathematics, Vol.1846, Springer-Verlag, Berlin (2004).

[3] H. Ammari, AND A. Khelifi, Electromagnetic Scattering by Small Dielectric Inhomogeinities.J. Math. Pures Appl. 82(2003), 749-842.

[4] H. Ammari, S. Moskow, And M. S. Vogelius , Boundary integral formulas for the reconstruction of electromagnetic imperfections of small diameter, ESAIM: Cont. Opt. Calc. Var., 9 (2003), 49-66.

[5] H. Ammari, AND D. Volkov, Correction of order three for the expansion of two dimensional electromagnetic fields perturbed by the presence of inhomogeinities of small diameter, J. Comput. Phys.,189 (2003),371-389.

[6] H. Ammari, M. S. Vogelius And D. Volkov, Asymptoticc formulas for perturbations in the electromagnetic fields due to the presence of inhomogeinities of small diameter II. The full Maxwell equations.J. Math. Pures Appl. 80(2001), 769-814.

[7] D. J. Cedio-Fengya, S. Moskow, And M. Vogelius, Identification of conductivity imperfections of small diameter by boundary measurements. Continuous dependence and computional reconstruction, Inverse Problems 14 (1998), 553-595.

[8] J. D. Cole, Perturbation Methods in Applied Mathematics, Blaisdell, Walthan, MA, 1968.

[9] A. Friedman And M. Vogelius, Identification of small inhomogeneities of extreme conductivity by boundary measurements: a theorem on continuous dependence, Arch. Rat. Mech. Anal. 105 (1989), 299-326.

[10] C. HazARD, AND M. LenOIR, On the solution of time-harmonic scattering problems for Maxwell's equations. SIAM J. Math. Anal. 27 (6)(1996), 1597-1630.

[11] A. M. IL'In, Matching of Asymptotic Expansions of Solutions of Boundary Value Problems, Translations of Mathematical Monographs, volume 102, Americain Mathematical Society, Providence, Rhode Island, 1992.

[12] A. B. Movchan And S. K. Serkov, The Pólya-Szegö matrices in asymptotic models of dilute media, Euro. Jnl of Appl. Math. 8 (1997), 595-621.

[13] J. C. NÉDÉLEC, Acoustic and Electromagnetic Equations. Integral Representation for Harmonic Problem, Springer-Verlag, New York, 2001.

[14] J. Sanchez Hubert and E. Sanchez Palencia, Vibration and Coupling of Continuous Systems, Springer-Verlag 1989.

[15] M. Vogelius And D. Volkov, Asymptotic formulas for perturbations in the electromagnetic fields due to the presence of inhomogeneities, Math. Model. Numer. Anal. 34 (2000), 723-748.

Christian DAVEAU, -Adresse: Département de Mathématiques, Site Saint-Martin II, BP 222, \& Université de Cergy-Pontoise, 95302 Cergy-Pontoise Cedex, France.

- Email: christian.daveau@math.u-cergy.fr

-Tel : (33) (0)1342566 72. -Fax : (33) (0)134256645.

Abdessatar KHELIFI, -Adresse: Département de Mathématiques, \& Université des Sciences de Carthage, Bizerte, 7021, Tunisie.

-Email: abdessatar.khelifi@fsb.rnu.tn

-Tel : (216) 975317 13. -Fax : (216) 72590566. 